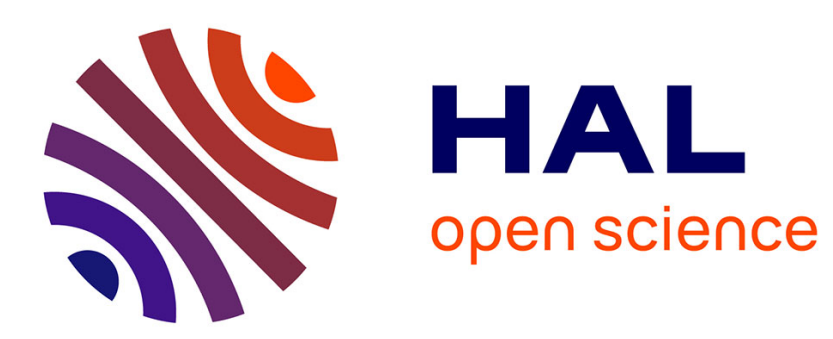

\title{
Identification du modèle thermique d'une machine à induction de puissance moyenne
}

Ch. Chillet, A.W. Hadi, R. Perret, R. Isnard

\section{To cite this version:}

Ch. Chillet, A.W. Hadi, R. Perret, R. Isnard. Identification du modèle thermique d'une machine à induction de puissance moyenne. Revue de Physique Appliquée, 1989, 24 (9), pp.923-931. 10.1051/rphysap:01989002409092300 . jpa-00246128

\section{HAL Id: jpa-00246128 https://hal.science/jpa-00246128}

Submitted on 1 Jan 1989

HAL is a multi-disciplinary open access archive for the deposit and dissemination of scientific research documents, whether they are published or not. The documents may come from teaching and research institutions in France or abroad, or from public or private research centers.
L'archive ouverte pluridisciplinaire HAL, est destinée au dépôt et à la diffusion de documents scientifiques de niveau recherche, publiés ou non, émanant des établissements d'enseignement et de recherche français ou étrangers, des laboratoires publics ou privés. 


\title{
Identification du modèle thermique d'une machine à induction de puissance moyenne
}

\author{
Ch. Chillet, A. W. Hadi, R. Perret et R. Isnard \\ Laboratoire d'Electrotechnique de Grenoble (U.A. C.N.R.S. 355), E.N.S.I.E.G., Institut National Polytech- \\ nique de Grenoble, B. P. 46, 38402 Saint Martin d'Hères, France
}

(Reçu le 9 janvier 1989, révisé le 20 avril 1989, accepté le 19 mai 1989)

\begin{abstract}
Résumé. - Dans cet article, les auteurs définissent un modèle thermique simple d'une machine asynchrone de puissance moyenne. Ils mettent en évidence les paramètres les plus sensibles ou les plus mal connus du modèle utilisé, et proposent une méthode d'identification de ces coefficients. Enfin, ils montrent des résultats validés par l'expérience.
\end{abstract}

\begin{abstract}
In this paper, the authors propose a simple thermal model of an induction machine of medium power. They insist on the best sensitive parameters and on the must unknown parameters of the model used, and they propose an identification method of these coefficients. Finally, they present results compared to experiments.
\end{abstract}

Dans des articles précédents $[1,2]$ nous avons présenté une modélisation thermique des machines à induction particulièrement bien adaptée aux moteurs de faibles puissances fermés et autoventilés. Les résultats obtenus étaient en bonne concordance avec l'expérience à condition de bien connaître certains paramètres d'échanges.

Dans le présent article nous voulons tester la validité du modèle dans le cas de machines de puissances plus importantes (jusqu'à quelques dizaines de $\mathrm{kW}$ ) et mettre en évidence les problèmes d'identification de paramètres qu'il faut résoudre pour obtenir une simulation précise de l'échauffement. Après avoir rappelé les principales caractéristiques de la méthode, et décrit brièvement la machine en test, nous définirons le modèle thermique le mieux adapté au cas étudié, puis nous proposerons une identification des paramètres sensibles mal connus et enfin, nous donnerons des résultats validés par l'expérience, en régime permanent et en régime transitoire.

\section{Rappel de la méthode utilisée.}

Le modèle utilise la technique de l'analyse nodale. La machine est découpée en blocs élémentaires isothermes définissant des domaines et des surfaces de séparation ; puis les domaines sont classés suivant le mode de transmission thermique (conduction, convection, rayonnement). Il faut ensuite déterminer les différents coefficients intervenant dans les équations de transfert et résoudre le système d'équations.

Pour vérifier et valider les études théoriques, nous disposons d'un banc de test. La machine étudiée est équipée de 8 sondes au stator, 8 sondes au rotor et d'un système de transmission sans contact. Les sondes sont des résistances au nickel dont on mesure les variations. Toute l'expérimentation est numérisée ; un micro-ordinateur effectue la saisie de mesure et calcule les températures correspondantes; les courbes d'échauffement en fonction du temps peuvent être directement tracées.

Nous avons choisi d'étudier l'échauffement d'une machine asynchrone à rotor bobiné, fermée et autoventilée car :

- géométrie simple ;

- possibilité d'injecter des pertes connues au stator et (ou) au rotor ; ceci permet de disposer d'un grand nombre d'essais pour valider le modèle ;

- la ventilation fait circuler l'air à l'extérieur de la machine sur les ailettes de refroidissement.

\section{Définition du modèle thermique}

Le choix du découpage de la machine en différents blocs élémentaires, résulte d'un compromis entre la 
simplicité du modèle (temps du calcul) et la qualité de l'information qu'il peut nous donner sur le comportement du système. Pour cela il est nécessaire de s'appuyer sur une bonne connaissance des propriétés thermiques de la machine afin d'en déduire les principaux chemins d'évacuation des pertes des éléments actifs vers l'extérieur.

1. Evacuation radiale: c'est sans nul doute le chemin le plus important. Le transfert des calories se fait à travers les circuits magnétiques rotorique et statorique et à travers l'entrefer.

2. Evacuation transversale : à partir du bobinage statorique, à travers les têtes de bobines et les cavités frontales et vers la carcasse.

3. Evacuation transversale : à partir du bobinage rotorique (machine à rotor bobiné), à travers les têtes de bobines et les cavités frontales et vers'la carcasse.

4. Evacuation transversale : à partir du fer rotorique, à travers l'arbre de la machine.

L'importance relative des différentes conductances intervenant le long du trajet, dépend des détails de construction de la machine, mais aussi de la taille de la machine. Un raisonnement élémentaire montre que si l'on fait une homothétie de rapport $k$ sur les dimensions de la machine, les conductances thermiques représentant un transfert par conduction croissent comme $k$, alors que les échanges par convection varient comme une surface et croissent en $k^{2}$. Cela veut dire, par exemple que l'importance des chemins 2 et 3 par rapport au chemin 4 augmente avec la puissance de la machine, et aussi que le long d'un chemin thermique pour une machine de faible puissance, ce sont les conductances « convectives» qui déterminent les chutes de température. Lorsque la taille de la machine augmente les variations de température aux bornes des conductances « conductives » deviennent de plus en plus importantes et le gradient de température dans les matériaux actifs n'est plus négligeable, on doit alors faire appel à des modèles plus sophistiqués en multipliant le nombre des blocs élémentaires ou en utilisant directement la méthode des éléments finis pour déterminer la répartition de température dans l'élément.

Pour fixer les idées nous avons représenté la répartition de température dans la machine que nous avons testée, le long de ces différents chemins thermiques, figure 1. Il s'agit de l'essai en charge nominale en alimentation sinusoïdale. L'observation de ces courbes montre que les chutes de température les plus importantes ont lieu pour le chemin principal à la surface extérieure de la machine et au niveau de l'entrefer, par contre les éléments actifs sont pratiquement isothermes. Il apparaît donc que les conductances que l'on doit déterminer avec le maximum de précision sont celles qui représentent :
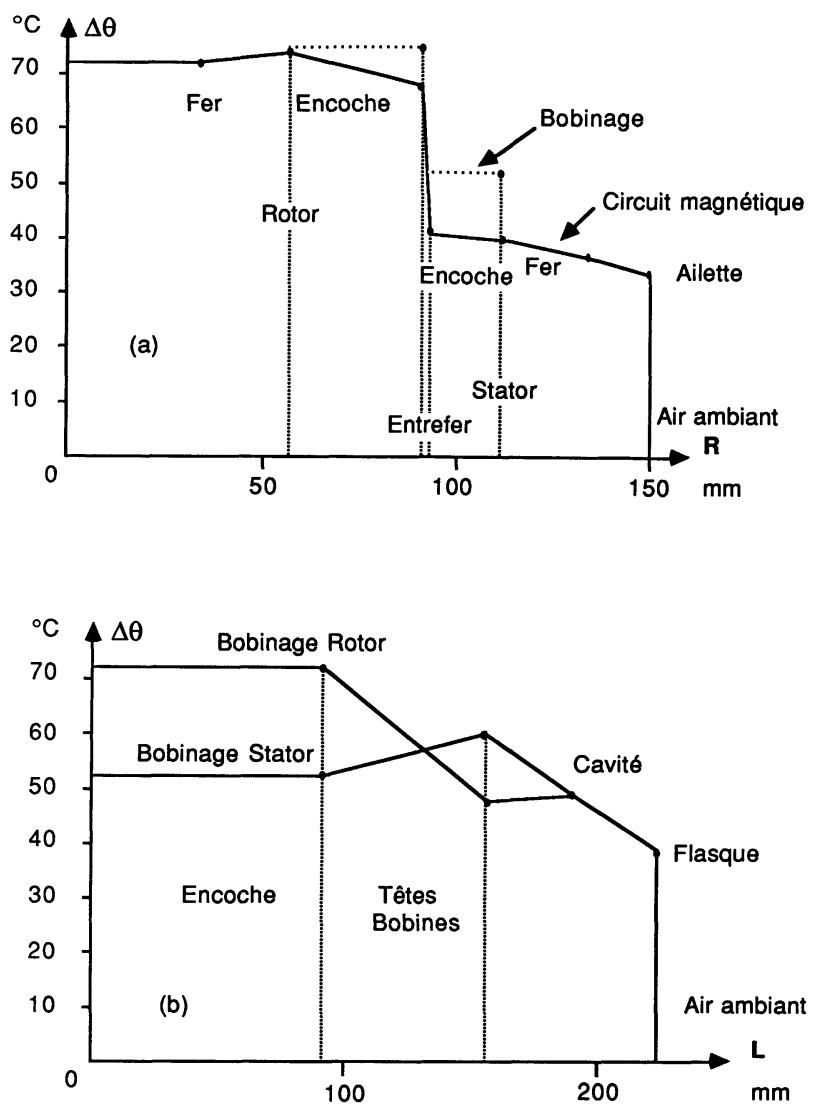

Fig. 1. - Distribution de la température dans la machine en charge nominale : a) dans la direction radiale ; b) dans la direction axiale.

[Temperature distribution in the machine under normal load ; a) radial direction ; b) axial direction.]

- les échanges entre la carcasse et l'air ambiant ;

- les échanges dans l'entrefer;

- les échanges entre le bobinage et le fer (rotor bobiné) ;

- les échanges entre le bobinage et le fer (stator) ;

- les échanges dans les cavités frontales;

- les échanges par l'arbre.

Les éléments du modèle retenu pour simuler le comportement thermique de la machine sont montrés dans les figures 2 et 3.

Les enroulements statoriques et rotoriques sont divisés en tête de bobine et conducteur dans l'encoche. Les circuits magnétiques statorique et rotorique sont également divisés en deux, les dents et la couronne. L'arbre est représenté par un élément correspondant à ses deux côtés mis en parallèle. La carcasse, les flasques avant et arrière sont regroupés en un seul élément ; ils constituent une enveloppe de la machine pratiquement isotherme.

Suivant notre terminologie, ce modèle est constitué de : 20 nœuds de liaisons, 15 éléments conductifs représentant les parties actives et conductives de la 


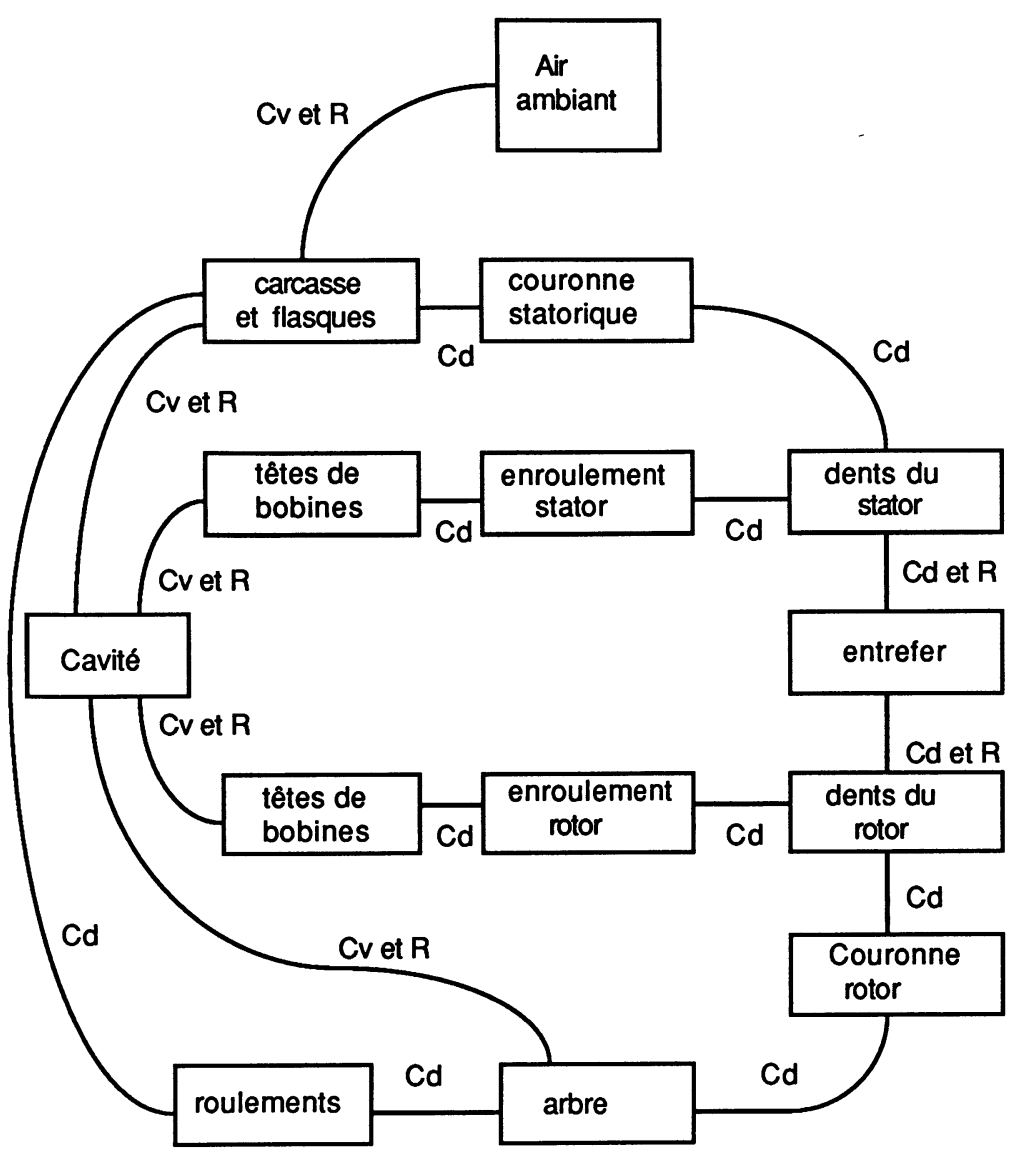

Fig. 2. - Eléments du modèle et modes de transfert thermique entre eux. Cd : conduction ; Cv : convection ; R : rayonnement.

[Elements of the model and their thermal exchange. $\mathrm{Cd}$ : conduction ; $\mathrm{Cv}:$ convection ; $\mathrm{R}$ : radiation.]

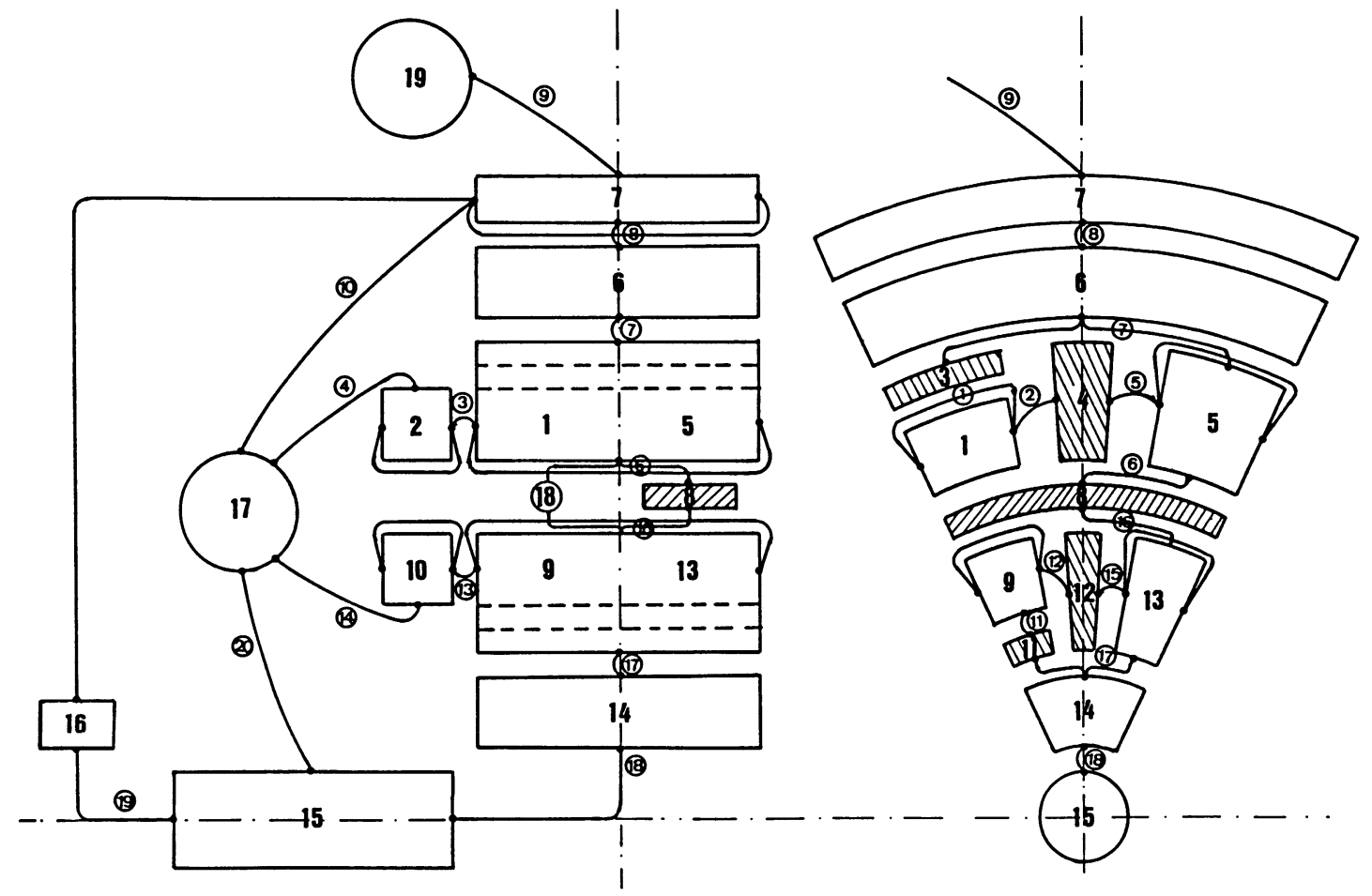

Fig. 3. - Modèle thermique de la machine.

[Thermal model of the machine.] 
machine, 1 élément convectif pour simuler l'échange dans les cavités à l'intérieur de la machine, 1 élément conductif et radiatif reflétant l'échange dans l'entrefer et 1 élément de référence qui est l'air ambiant. Ces éléments sont représentés sur la figure 3 par :

1 et 9 enroulements statoriques et rotori2 et 10 ques ;

têtes de bobines statoriques et rotoriques ;

5 et 13 dents statoriques et rotoriques ;

6 et 14 couronnes statoriques et rotoriques ;

7 carcasse et flasques ;

15 arbre ;

16 roulements ;

$3,4,11,12$ isolants ;

8 et 18 entrefer;

17 cavité ;

19 air ambiant.

Le découpage en blocs isothermes, ainsi que les connexions thermiques entre les différents éléments étant précisés, il faut déterminer les propriétés thermiques des éléments (conductivité, inertie thermique) à partir des données du constructeur ou directement par la mesure. Certaines conductances ne peuvent être qu'approchées par le calcul, il sera nécessaire d'ajuster les valeurs expérimentalement sur la base d'essais bien spécifiques où la répartition des pertes est connue sans ambiguïté.

Parmi les conductances mal connues on peut citer :

1) l'échange entre le milieu extérieur et la machine ;

2) l'échange thermique dans l'entrefer : plusieurs mécanismes d'échanges sont à envisager : convection naturelle, brassage de l'air sous l'effet de la rotation, conduction et rayonnement. Les nombres de Grashof et de Taylor calculés pour l'air de l'entrefer [14, 15 ], nous indiquent que seuls les deux derniers types d'échange sont à considérer. Dans notre plage de température, la conductance thermique totale de l'entrefer $G_{\mathrm{E}}$ peut se représenter avec une bonne précision, en se limitant au premier ordre, par :

$$
G_{\mathrm{E}}=G_{\mathrm{E} 0}(1+a \theta)
$$

où $\theta$ est la température de l'air en degré Celsius et $a$ un coefficient $\left(a=3,67 \times 10^{-3} \mathrm{~K}^{-1}\right)$.

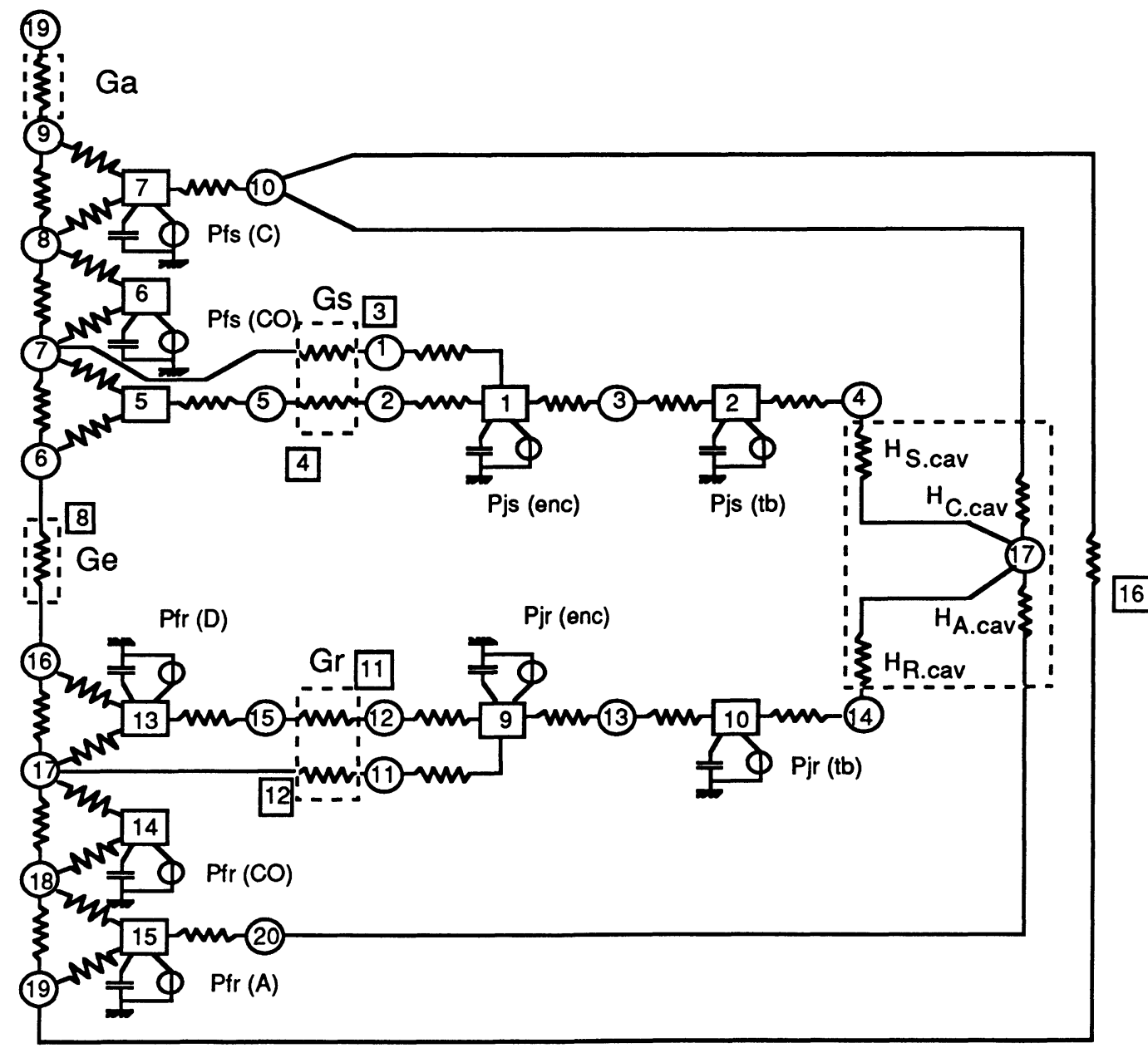

Fig. 4. - Schéma équivalent du modèle thermique de la machine (représenté Fig. 3).

[Equivalent representation of the thermal model of the machine (represented in Fig. 3).] 
Actuellement, la méthode que nous employons pour le modèle thermique, ne permet pas de faire varier les coefficients. On doit donc choisir le coefficient en fonction de la température finale estimée ;

3) échanges entre le bobinage et le circuit magnétique ;

Le calcul de la conductance de l'isolation est illusoire car on connaît mal l'épaisseur des différents isolants, du vernis et de l'air. Seul un essai correspondant à un échange localisé (pertes Joule et machine non ventilée) permet d'obtenir un résultat significatif ;

4) échanges dans les cavités frontales.

Le coefficient d'échange dans les cavités contenant les têtes de bobines peut être approché par un calcul simplifié. La topologie de cette cavité fait apparaître 4 échanges par rayonnement $\left(h_{\mathrm{S}-\mathrm{C}}, h_{\mathrm{R}-\mathrm{s}}, h_{\mathrm{R}-\mathrm{C}}, h_{\mathrm{A}-\mathrm{C}}\right)$ et 4 échanges par convection avec l'air ambiant $\left(h_{\mathrm{S}-\mathrm{a}}, h_{\mathrm{C}-\mathrm{a}}, h_{\mathrm{R}-\mathrm{a}}, h_{\mathrm{A}-\mathrm{a}}\right)$. Nous avons utilisé, pour ces calculs, les relations d'échange dans les espaces libres. Pour simplifier la résolution, on transforme ceci en un schéma équivalent étoile, ce qui fait apparaître un élément fictif : la cavité. Les conductances se résument alors à $H_{\mathrm{A} \text {-cav }}, H_{\mathrm{R} \text {-cav }}, H_{\mathrm{S} \text {-cav }}$, $H_{\text {C-cav }}$ ( $(\mathrm{S}:$ stator, $\mathrm{R}$ : rotor, $\mathrm{A}:$ arbre, $\mathrm{C}:$ carcasse, a : air, cav : cavité).

\section{Identification du modèle.}

Les considérations du paragraphe précédent permettent de définir les conductances les plus importantes. Il s'agit maintenant d'en ajuster la valeur à partir d'essais particuliers.

Sur la figure 4, nous avons représenté les conductances et les capacités calculées par le modèle et représentant les éléments dont l'ajustement des valeurs des conductances n'a que peu d'importance puisqu'elles concernent des zones où le gradient de température est faible, ainsi que les conductances à identifier encadrées par un pointillé.

A. Pour fixer les conditions d'échange entre la machine et le milieu extérieur et assurer une température homogène de la carcasse, nous avons placé la machine dans un conduit, à l'intérieur duquel un ventilateur indépendant fait circuler l'air à vitesse constante. Dans ces conditions, on peut relever l'échauffement de la surface de la machine, en régime permanent, en fonction des pertes totales. $\mathrm{La}$ courbe obtenue peut se représenter par une droite (Fig. 5) dont la pente est égale au coefficient d'échange. La conductance thermique $\mathrm{Ga}$ est alors déterminée et vaut pour tous les essais $43 \mathrm{~W} \cdot \mathrm{K}^{-1}$.

B. Les conductances des isolations du bobinage et du circuit magnétique ne sont pas déterminées avec suffisamment de précision à partir des essais de la machine à l'arrêt et alimentée en courant continu au

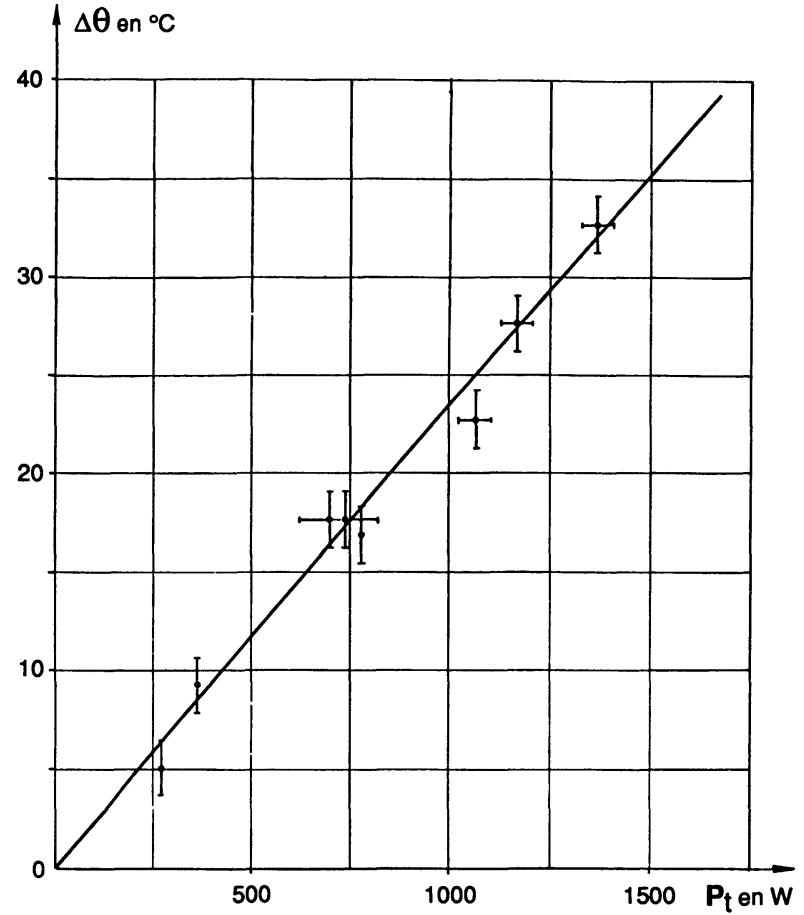

Fig. 5. - Echauffement de la surface extérieure de la machine en fonction des pertes totales.

[External surface heating of the machine as a function of the total losses.]

stator et au rotor. Nous avons dû leur adjoindre l'essai en court-circuit qui est aussi un essai où les échanges avec la cavité sont faibles (vitesse nulle) et où la répartition des pertes est connue avec une précision acceptable.

En utilisant le modèle pour ajuster les écarts de température bobinage/circuit magnétique, en jouant sur les conductances thermiques des isolations de bobinage et en nous plaçant dans l'hypothèse ou le calcul des échanges dans la cavité est correct, nous avons obtenu :

$$
G_{\mathrm{S}}=30,8 \mathrm{~W} \cdot \mathrm{K}^{-1} \text { et } G_{\mathrm{R}}=43,8 \mathrm{~W} \cdot \mathrm{K}^{-1} .
$$

C. La conductance thermique de l'entrefer dépend directement de la surface équivalente de l'entrefer. Or la valeur de cette surface est difficile à déterminer compte tenu de la géométrie compliquée de la machine (becs et cales d'encoche). Les résultats de l'essai en court-circuit nous ont permis d'ajuster la valeur de $G_{\mathrm{E} 0}$. La surface équivalente d'entrefer est alors légèrement plus grande que la surface du circuit magnétique.

$$
G_{\mathrm{E} 0}=4,54 \mathrm{~W} \cdot \mathrm{K}^{-1} .
$$

D. Les conductances thermiques représentant les échanges dans les cavités frontales dépendent fortement de la vitesse de rotation de la machine. Elles 
sont ajustables à partir des essais de refroidissement à différentes vitesses de rotation qui sont des essais où les pertes sont faibles et correctement localisées. Toutefois, pour qu'une identification de conductance à partir d'essais en régime transitoire soit valable, il est nécessaire de connaître les inerties thermiques des éléments. Pour palier à la mauvaise détermination de ces termes, due à une part d'arbitraire dans le découpage des éléments ou tout simplement à une méconnaissance de certains détails de construction, nous avons adopté la procédure décrite ci-dessous.

Dans un premier temps, à partir des essais de refroidissement à l'arrêt et en utilisant les valeurs des conductances précédemment déterminées ainsi que les valeurs des conductances d'échange dans la cavité données par le calcul, nous avons ajusté les courbes du modèle à celles de l'expérience, en modifiant légèrement les inerties des différents éléments.

Dans un deuxième temps, à partir de l'essai de refroidissement à 1500 tr.min ${ }^{-1}$ où l'on connaît les inerties, il est possible d'ajuster les courbes du modèle à celle de l'expérience. L'ajustement doit se faire sur le départ des courbes car le modèle ne tient pas compte de la variation de la conductance de l'entrefer en fonction de la température. Les valeurs obtenues pour une vitesse de $1500 \mathrm{tr} . \mathrm{min}^{-4}$ sont les suivantes :

$$
\begin{array}{ll}
H_{\mathrm{S} \text { cav }}=4,7 \mathrm{~W} \cdot \mathrm{K}^{-1}, & H_{\mathrm{R}-\mathrm{cav}}=9,5 \mathrm{~W} \cdot \mathrm{K}^{-1} \\
H_{\mathrm{C} \text {-cav }}=33,3 \mathrm{~W} \cdot \mathrm{K}^{-1}, & H_{\mathrm{A}-\mathrm{cav}}=9,6 \mathrm{~W} \cdot \mathrm{K}^{-1} .
\end{array}
$$

\section{Comparaison avec l'expérience.}

La confrontation des résultats de l'expérience et du modèle doit se faire uniquement avec les essais où les pertes sont localisées avec certitude. Lorsque ce n'est pas le cas, on dispose en effet d'un paramètre supplémentaire, le pourcentage de perte à attribuer à un élément, qui permettra toujours d'ajuster plus ou moins la réponse du modèle; ces essais ne pourront donc pas être utilisés pour justifier la validité du modèle.

Les températures importantes qui caractérisent l'état thermique de la machine et qui sont mesurées expérimentalement sont au nombre de cinq: les températures des bobinages statorique et rotorique (dans l'encoche), les températures des circuits magnétiques statorique et rotorique et la température des ailettes extérieures. La machine se comporte donc du point de vue thermique comme un système à cinq sorties (les températures) et à six entrées (les pertes dans les éléments).

Nous donnons (Figs. 6 et 7) l'évolution des températures de deux points tests (bobinage statorique et rotorique) dans les essais en court-circuit (a), alimentation en courant continu au stator (b) puis au rotor (c), et refroidissement à l'arrêt (d). Le comportement à vitesse variable du modèle est illustré par la figure 8 représentant l'essai de refroidissement à $600 \mathrm{tr} / \mathrm{min}$. Pour cet essai, nous utilisons les coefficients d'échanges dans la cavité identifiés dans l'essai à $1500 \mathrm{tr} / \mathrm{min}$ affectés d'un coefficient $(\mathrm{N} / 1500)^{0,7}$.

Les courbes expérimentales tracées en pointillés ont été obtenues en moyennant les relevés expérimentaux. On constate en effet sur les véritables courbes une dispersion de $\pm 1,5 \mathrm{~K}$ due aux différents bruits recueillis au niveau des sondes ou introduits par la chaîne de mesure (pas de quantification de $0,7 \mathrm{~K})$. Les écarts constatés sont à l'intérieur de cette marge d'incertitude. Sur l'essai de refroídissement, les écarts de température ne tendent pas vers 0 ; ce phénomène est attribuable au dispositif de transmission des températures rotoriques qui dissipe constamment environ $50 \mathrm{~W}$. Nous avons pris en compte grossièrement ce phénomène dans le modèle, en injectant une puissance de $25 \mathrm{~W}$ à l'extrémité de l'arbre.

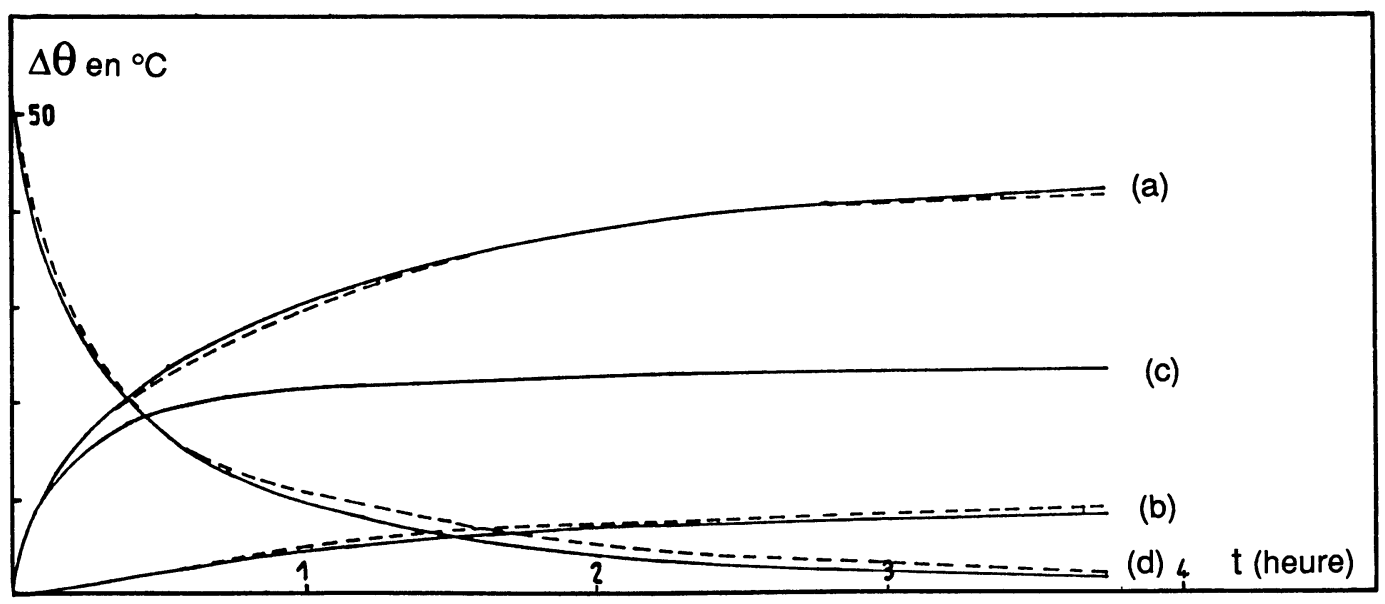

Fig. 6. - Evolution de la témpérature du bobinage statorique.

[Temperature evolution of stator winding.] 


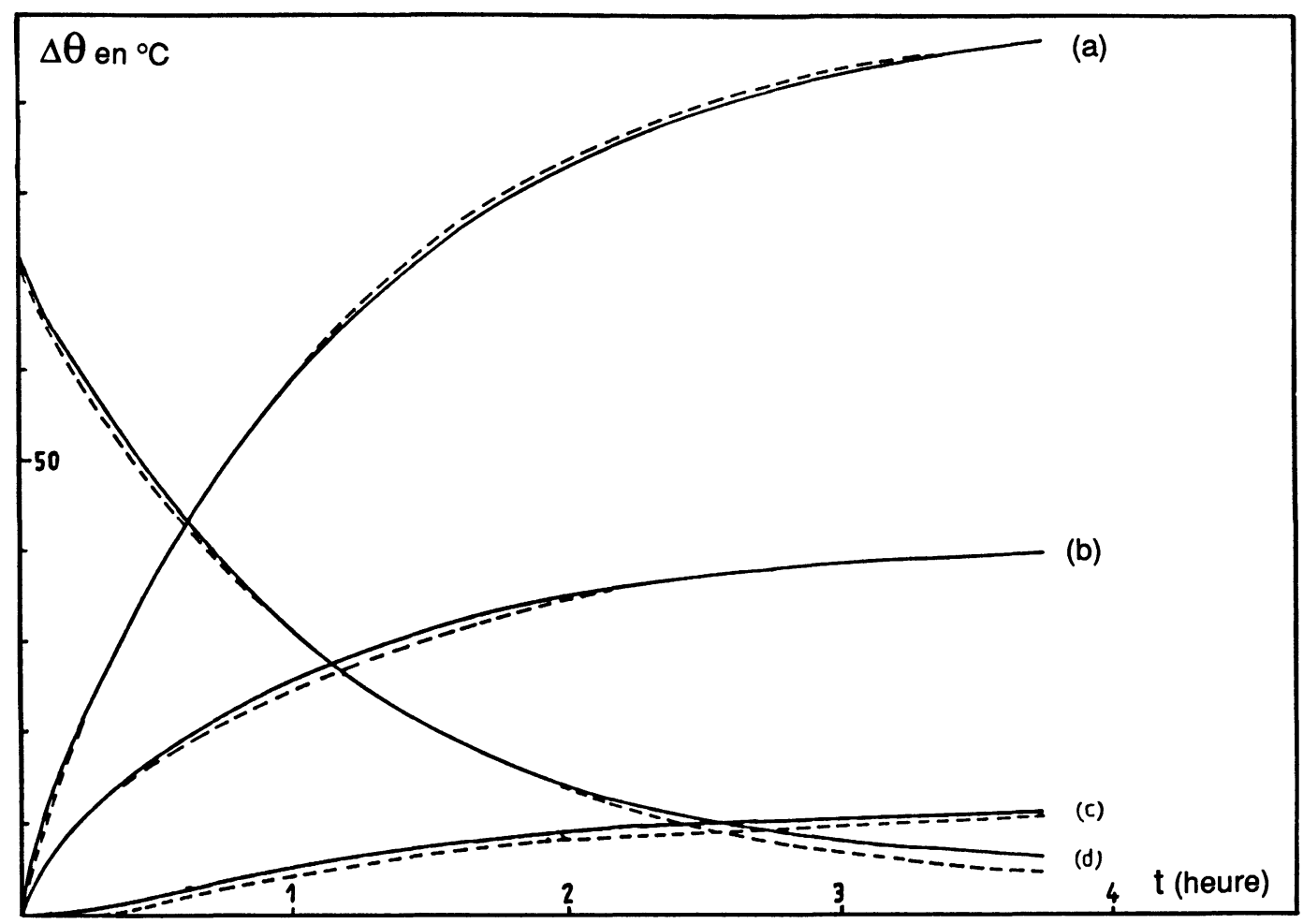

Fig. 7. - Evolution de la température du bobinage rotorique.

[Temperature evolution of rotor winding.]

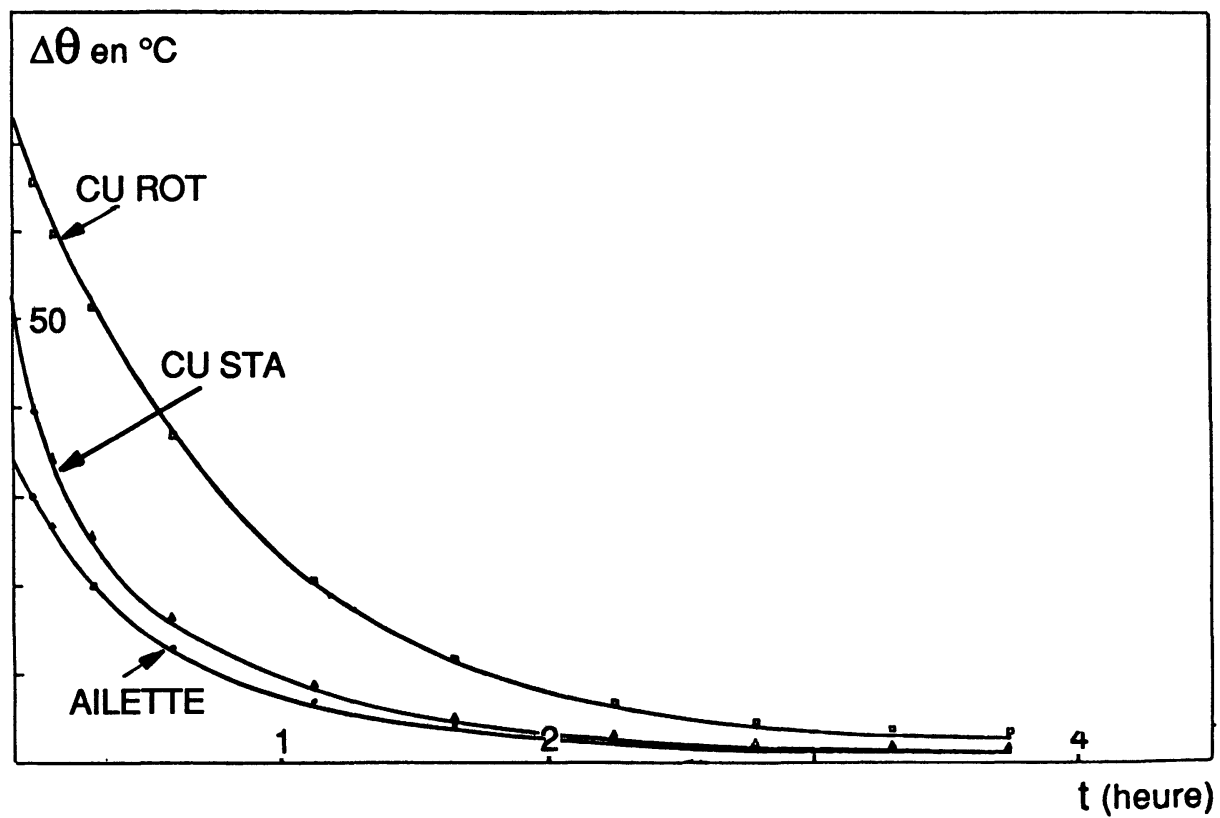

Fig. 8. - Essai de refroidissement à $600 \mathrm{tr} / \mathrm{min}$.

[Cooling test at $600 \mathrm{rpm}$.]

\section{Conclusion.}

Nous avons présenté une méthode permettant d'établir un modèle thermique simple d'une machine à induction. Dans ce modèle, basé sur le principe de l'analyse nodale, on divise la machine en blocs élémentaires. La qualité de cette représentation est très fortement liée à l'évaluation des paramètres d'échange entre blocs. C'est pourquoi nous avons recherché des essais caractéristiques pour identifier 
les paramètres les plus sensibles ou les plus mal connus. Cette méthode de détermination a donné des résultats très satisfaisants que ce soit en régime statique ou en régime dynamique.

Nous avons montré qu'un découpage grossier de la machine était suffisant pour appréhender les écarts de température globaux. Si l'identification est bien conduite, le modèle obtenu est un reflet très fidèle de la réalité. Plusieurs utilisations sont alors possibles :

- conception de machines nouvelles voisines de la machine étudiée ou comportant des parties analogues, en utilisant les conductances moyennes entre éléments déterminées par le modèle ;

- détermination et localisation des pertes dans une machine.

\section{Annexe A.}

Relations d'échange entre surfaces tournantes (entrefer).

La conductance de l'entrefer $[8,14,15]$ peut s'écrire de la façon suivante :

$$
G_{\mathrm{E}}=h_{\mathrm{e}} \cdot S_{\mathrm{e}} \quad \text { avec } \quad h_{\mathrm{e}}=k \cdot e^{-1} \cdot \mathrm{Nu} .
$$

Le nombre de Nüsselt est donné dans le tableau suivant en fonction du nombre de Taylor.

\begin{tabular}{|c|l|}
\hline \multicolumn{1}{|c|}{$\mathrm{Ta}$} & \multicolumn{1}{|c|}{$\mathrm{Nu}$} \\
\hline $0<\mathrm{Ta}<41$ & $\mathrm{Nu}=1$ \\
\hline $41<\mathrm{Ta}<100$ & $\mathrm{Nu}=0,106 \cdot \mathrm{Ta}^{0,63} \cdot \mathrm{Pr}^{0,27}$ \\
\hline $100<\mathrm{Ta}<(\mathrm{Ta})_{\mathrm{v} \cdot \mathrm{t}}$ & $\mathrm{Nu}=0,193 \cdot \mathrm{Ta}^{0,5} \cdot \mathrm{Pr}^{0,27}$ \\
\hline
\end{tabular}

$\operatorname{Re}=\left[\rho \cdot \mu^{-1}\right]_{\mathrm{m}} \cdot e \cdot R \cdot \Omega \mathrm{Ta}=\operatorname{Re}_{\mathrm{m}} \cdot[e / R]^{0,5}$.

$(\mathrm{Ta})_{\mathrm{v}, \mathrm{t}}$ est le nombre de Taylor correspondant à la vitesse critique donnée par: $\operatorname{Re}=41,1 \cdot[R / e]^{0,5}$ et à partir de laquelle l'écoulement turbulent devient prépondérant.

Echanges entre air et surface tournante (disque ou cylindre).

L'importance de la convection par rapport à l'effet de la rotation $[3,8,14]$ est donnée par le paramètre $Y$ :

$$
Y=\operatorname{Re} \cdot \mathrm{Gr}^{-0,5}
$$

avec

$$
\begin{aligned}
& \mathrm{Gr}=\left[\beta \cdot \rho^{2} \cdot \mu^{-2} \cdot g\right]_{\mathrm{m}} \cdot D^{3} \cdot \Delta T \\
& \operatorname{Pr}=\left[\rho \cdot \mathrm{Cp} \cdot k^{-1}\right]_{\mathrm{m}} .
\end{aligned}
$$

Pour des valeurs de $Y<0,2$ les effets de la rotation sont négligeables et l'échange est le même que dans le cas du cylindre stationnaire. Lorsque $Y>0,2$ et $P r<1$ (cas de l'air), le nombre de Nüsselt prend la valeur suivante.

$$
\mathrm{Nu}=0,1075 \cdot(\mathrm{Gr} \cdot \mathrm{Pr})^{0,35} \cdot\left(1+0,5 \cdot Y^{2}\right)^{0,35} .
$$

Cette formule a été vérifiée pour $10^{3}<\operatorname{Re}<5 \times$ $10^{6}$ et $5 \times 10^{6}<\mathrm{Gr} \cdot\left[1+0,5 \times \mathrm{Y}^{2}\right]<10^{11}$.

Echanges par rayonnement entre surfaces concentriques proches ou deux surfaces parallèles (cas de l'entrefer).

Le paramètre d'échange prend la valeur suivante dans le cas de deux surfaces de même nature [3] :

$$
\begin{aligned}
\mathrm{hr}=4 \cdot \varepsilon \cdot \sigma \cdot(2-\varepsilon)^{-1} \cdot T^{3} & \times \\
& \times\left[1+(\Delta T /(2 \cdot T))^{2}\right] .
\end{aligned}
$$

\section{Abréviations utilisées dans l'annexe.}

$\varepsilon=$ coefficient d'émissivité des deux surfaces, $\sigma=$ constante de Stefan-Boltzman,

$T=$ température de l'air en $\mathrm{K}, \Delta T=$ écart de température entre surface,

$R=$ rayon du rotor, $e=$ épaisseur de l'entrefer,

$\boldsymbol{\Omega}=$ vitesse angulaire, $\rho=$ masse volumique de l'air, $\mu=$ viscosité dynamique,

$k$ = conductivité thermique, $S_{\mathrm{e}}=$ surface de l'entrefer

$\mathrm{Cp}=$ chaleur massique de l'air, $\beta=$ coefficient de dilatation,

$g=$ accélération de la pesanteur, $D=$ diamètre, $m=$ température moyenne

\section{Annexe B.}

Caractéristiques électriques de la machine asynchrone étudiée :

Puissance : $11 \mathrm{~kW}$.

Stator : tension $=220 / 380 \mathrm{~V}$ courant $=40,7 / 23,5 \mathrm{~A}$.

Rotor : tension $=280 \mathrm{~V}$ courant $=25 \mathrm{~A}$.

Fréquence $=50 \mathrm{~Hz}$, vitesse de rotation $=1450 \mathrm{tr} \cdot \mathrm{min}^{-1}$.

Facteur de puissance $n=0,82$, rendement $n=0,87$.

Caractéristiques géométriques :

Circuit magnétique rotorique : longueur $184 \mathrm{~mm}$, diamètre extérieur $184 \mathrm{~mm}$.

Circuit magnétique statorique : diamètre intérieur $185 \mathrm{~mm}$, extérieur $270 \mathrm{~mm}$.

Nombre d'encoches au stator 36, au rotor 24 . 


\section{Bibliographie}

[1] Roye D., Perret R., Définitions des règles de modélisation thermique des machines électriques tournantes, Revue Phys. Appl. 20 (1985).

[2] Roye D., Perret R., Modélisation thermique d'une machine à induction, R.G.E. (juillet 1985).

[3] Hadi A. W., Etude de.l'échauffement d'un moteur asynchrone à rotor bobiné, Thèse de docteur de l'INP Grenoble (mai 1987).

[4] Roye D., Modélisation thermique des machines tournantes. Application à la machine à induction, Thèse de docteur d'état INP Grenoble (novembre 1983).

[5] MaRTy M., Contribution à l'étude de l'échauffement des machines électriques tournantes, Thèse de docteur d'état INP Toulouse (avril 1965).

[6] Chalbi O., Contribution à l'étude des pertes harmoniques dans les machines à induction, Thèse de docteur ingénieur INP Grenoble (1968).

[7] Eckert E. R. G., Drake R. M., Analysis of heat and mass transfert (Inter Student Edition, McGraw-Hill Book Co., Inc.) 1972.

[8] KAYS W. M., BJORKLUND I. S., Heat transfer from a rotating cylinder with and without crossflow, Trans. A.S.M.E. 80 (1958) 70-78.
[9] SAY M. G., Alternating current machines (Fifth Edition Pitman London) 1983.

[10] Baнbouth S., Etude en régime permanent d'une machine asynchrone alimentée par un onduleur à transistors ; performances, pertes, échauffements, Thèse de docteur ingénieur INP Grenoble (octobre 1981).

[11] Olivier E., Etude des performances d'un moteur asynchrone à cage alimenté par un onduleur à transistors Mos de puissance commandé en modulation de largeur d'impulsion, Thèse de $3^{\circ}$ cycle INP Grenoble (octobre 1982).

[12] BEN-NASR, Contribution à l'étude de l'échauffement des machines asynchrones alimentées par commutateur de courant, Thèse de $3^{e}$ cycle INP Grenoble (novembre 1984).

[13] Carslaw H. C., Jeager J. C., Conduction of heat in solids (second edition, Oxford Press) 1959.

[14] Heat Transfer and Fluid Flow Data Book (General Electric Compagny Schenectady, New York).

[15] GAZleY C., Heat transfer characteristics of the rotational and axial flow between concentric cylinder, Trans. A.S.M.E. 80 (1958) 79-90. 